\title{
Dynamic water allocation policies improve the global efficiency of storage systems
}

\author{
Amin Niayifar ${ }^{1}$ and Paolo Perona ${ }^{2}$ \\ 1 Stream Biofilm and Ecosystem Research Laboratory, Institute of Environmental Engineering, EPFL-ENAC, \\ Lausanne, Switzerland; amin.niayifar@epfl.ch \\ 2 Institute for Infrastructure and Environment, School of Engineering, The University of Edinburgh, Edinburgh, UK; \\ paolo.perona@ed.ac.uk
}

\begin{abstract}
Water impoundment by dams strongly affects the river natural flow regime, its attributes and the related ecosystem biodiversity. Fostering the sustainability of water uses e.g., hydropower systems thus implies searching for innovative operational policies able to generate Dynamic Environmental Flows (DEF) that mimic natural flow variability. The objective of this study is to propose a Direct Policy Search (DPS) framework based on defining dynamic flow release rules to improve the global efficiency of storage systems. The water allocation policies proposed for dammed systems are an extension of previously developed flow redistribution rules for small hydropower plants by Razurel et al. (Water resources management, 30, 207-223 (2016)).The mathematical form of the Fermi-Dirac statistical distribution applied to lake equations for the stored water in the dam is used to formulate non-proportional redistribution rules that partition the flow for energy production and environmental use. While energy production is computed from technical data, riverine ecological benefits associated with DEF are computed by integrating the Weighted Usable Area (WUA) for fishes with Richter's hydrological indicators. Then, multiobjective evolutionary algorithms (MOEAs) are applied to build ecological versus economic efficiency plot and locate its (Pareto) frontier. This study benchmarks two MOEAs (NSGA II and Borg MOEA) and compares their efficiency in terms of the quality of Pareto's frontier and computational cost. A detailed analysis of dam characteristics is performed to examine their impact on the global system efficiency and choice of the best redistribution rule. Finally, it is found that non-proportional flow releases can statistically improve the global
\end{abstract}


efficiency, specifically the ecological one, of the hydropower system when compared to constant minimal flows.

Keywords: Dynamic environmental flows, Non-proportional water allocation, Hydropower, NGSA II optimization, Fish habitat indicators, Richter's hydrological indicators

\section{Introduction}

The practice of impounding water from mountain streams for anthropogenic uses has been shown to possibly affect - notably to reduce - the biodiversity of riverine ecosystems (Assani et al., 2010, Kennard et al., 2010, Kern et al., 2011, Konar et al., 2013). The biogeomorphological basis responsible for such an effect is related to the establishment of minimal constant discharges from river intakes and/or reservoirs (Arthington et al., 2006). In Switzerland, for example, this static rule is regulated by Swiss Federal Legislation and corresponds to the release of a constant (or seasonally constant) flow rate, $Q_{347}$. This value is close to the flow quantile exceeded on average $95 \%$ of the time, which is obtained from the flow duration curve of the natural flow regime (e.g., Franchini et al., 2011). Many countries have adopted this ecological measure because of its simplicity. An example of the application of the constant minimal flows that modifies a natural flow regime is shown in the hydropower scheme of Figure 1, where much of the annual runoff volume is stored in the dam and allocated as flowrate, $Q_{\text {hydro }}(t)$, to satisfy energy demand. The flow rate allocated to the environment, $Q_{\text {env }}(t)$ based on a minimal flow policy shows almost constant river discharge with the exception of some peaks. The peaks are due to both uncaptured runoff or storage releases to the environment when the maximum capacity of the reservoir is reached during flood events (Schweizer et al., 2007, Petts, 2009). 


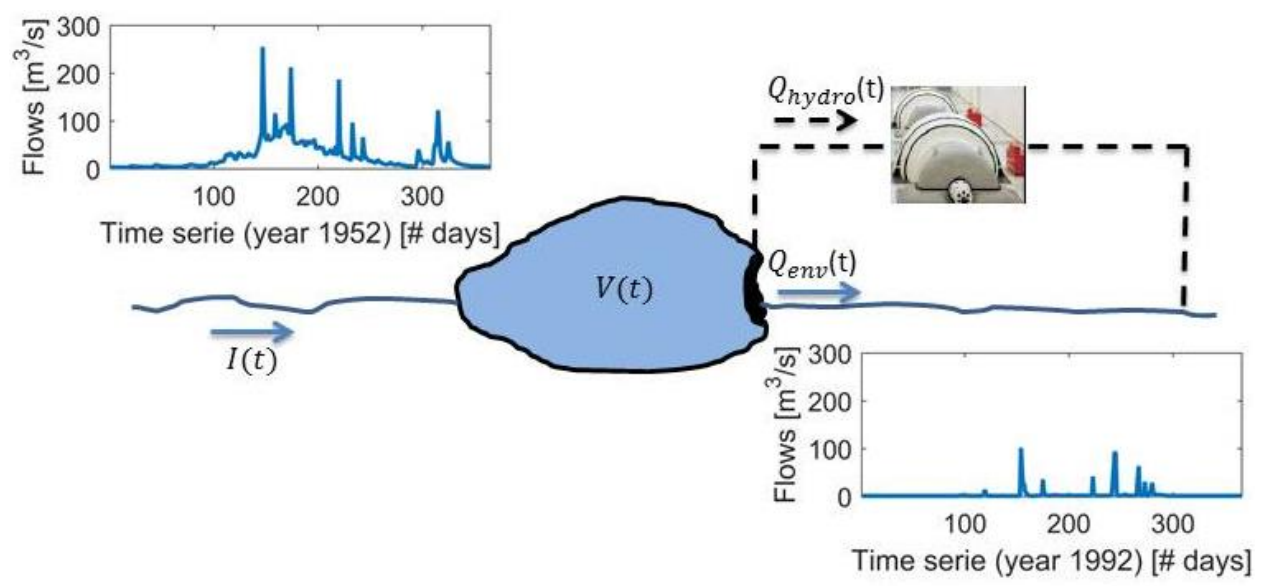

Figure 1. Schematic of the dammed systems. Hydrographs represent the daily flow rate of Maggia river before (1952) and after (1992) installation of the dam.

Ultimately, although favorable for certain aquatic species, the application of minimal flow policies tend to "homogenize" river hydrographs, and produces similar long-term effects even for ecosystems in very different geographic locations (Arthington et al., 2006, Moyle and Mount, 2007).

Extensive research has been performed on reservoirs water management and optimization (e.g., Oliveira and Loucks, 1997, Cui and Kuczera, 2005). In these works, the best operating rules for storage systems are chosen to optimize one or more objectives. Operating policies usually determine the release rule (e.g., discharge or dam storage) for the reservoir at any time step. In the literature, different methods have been proposed to define efficient operational policies. Dynamic Programming (DP) and its extension, Stochastic Dynamic Programming (SDP), have been widely used in the literature (e.g., Yeh, 1985, Castelletti et al., 2008) to define efficient operational policies in storage systems. These techniques improve the operational efficiency of storage systems, but their application is limited (Giuliani et al., 2015) because of problem dimensionality (Bellman, 1957), modeling parameters versus data availability (Tsitsiklis and Van Roy, 1996) and representation of multiple objectives (Powell, 2007).

Direct policy search (DPS) methods are a viable alternative to overcome the three shortcomings of DP and SDP (e.g., Dariane and Momtahen, 2009, Guo et al., 2012). DPS methods parametrize the operational policy using a predefined parametric family of functions and optimize it based on the objectives of the studied reservoir (Giuliani et al., 2015). The choice of defining operational 
policies is usually performed by defining some empirical and practical approaches. Some recent works (e.g., Salazar et al., 2016) have tried to generalize the definition of operational rules using nonlinear approximating networks (e.g., artificial neural networks and radial basis functions). For the optimization approach used in the DPS methods, gradient based and Evolutionary Algorithms (EAs) have been extensively used to find efficient operational rules for reservoir systems. Particularly, EAs have shown better efficiency in handling the performance uncertainties compared to methods based on predicting absolute performance or performance gradient (Heidrich-Meisner and Igel, 2008). Several studies have investigated the performance of methods for optimizing operational rules for reservoir systems (e.g., Salazar et al., 2016).

As discussed before, the goal of defining operational rules for reservoir systems is to optimize their operational efficiency based on the characterization of some objectives. Depending on the function of each reservoir system, several objectives have been considered in the literature, such as electricity production, irrigation, potable water supply, and flood protection. Substantial improvement in the efficiency of reservoir with respect to the considered objectives was achieved (e.g., Cui and Kuczera, 2005, Dariane and Momtahen, 2009). The riverine ecosystem is acknowledged to be significantly affected by reservoir operations due to the alteration of the natural flow regime. However, minimizing the related environmental impact has not been considered as a detailed and well-focused objective in the field of defining operational rules for the reservoirs. The primary goal of this study is to develop a new DPS framework by defining a new class of functions (i.e., non-proportional flow release) for reservoir operational rules, whose environmental impact is comprehensively assessed and minimized while maintaining the economical (i.e., energy production) efficiency.

Efforts to summarize existing frameworks and guidelines for determining environmental flows have been recently proposed (Petts, 1996, Poff et al., 2010, Meijer et al., 2012). It is generally accepted that future ecologically sustainable exploitation of water resources in dammed systems requires seeking innovative operational flow release strategies that mimic the natural flow regime. This challenging aspect concerns with the ability to find new dynamic environmental flows that can improve ecological efficiency with respect to constant minimal flow policies (e.g., Arthington et al., 2006, Bartholow, 2010, Bizzi et al., 2012) while maintaining economic benefit. Perona et al. (2013) have introduced the idea of engineering Dynamic Environmental Flows 
(henceforth referred to as DEFs) releases by considering the riparian environment as a nontraditional water use. Increasing hydropower production without straining the environment has then shown to be feasible at least for water systems without storage such as small hydropower (e.g., Perona et al., 2013, Lazzaro et al., 2013, Gorla and Perona, 2013, Ta et al., 2016, Razurel et al., 2016). Gorla (2014) and Razurel et al. (2016) have generalized the method by introducing the concept of non-proportional redistribution. In this work, we intend to show that the nonproportional redistribution concept is also applicable to traditional dammed systems, and leads to Pareto efficient solution containing non-proportional policies. Compared to the case of small hydropower, dammed systems have storage dynamics that require multiobjective dynamic programming numerical approaches. These can be computationally heavy when thousands of policies have to be simulated. Hence, we use optimization methods (NSGA II and Borg MOEA) to speed up the numerical process and build the efficiency plot. Furthermore, the results of the Borg MOEA and NSGA II are compared in terms of computational cost and fitness of Pareto's frontier to find the efficient optimization method. Eventually, DEFs releases obtained from nonproportional redistribution rules are found to steer future water resources management towards ecosystem functioning and sustainability.

\section{Methodology}

We tackle the problem of finding Pareto-efficient both ecological and economical operational rules for dammed systems by simulating state-dependent non-proportional flow redistribution rules. Ecological benefits for the riverine corridor due to DEFs, are obtained by aggregating the fish habitat suitability indexes (HSI) and Richter's hydrological indicators. We use multiobjective evolutionary algorithms (MOEAs) to build the Pareto's frontier as a computationally efficient alternative to direct simulation of high number of selected strategies. The use of MOEAs guarantees that solutions lying on the frontier satisfy both maximal power production and ecological sustainability. Moreover, this method can be implemented in a graphical user interface form for practical use by stakeholders and water managers. We begin by introducing non-proportional flow redistribution. 


\subsection{Non-proportional flow redistribution}

The schematic of a dammed system for hydropower production is shown in Figure 1 where the following expression represents the reservoir continuity equation governing stored water volume dynamics at each time step $t$ :

$$
\frac{d V(t)}{d t}=I(t)-Q_{e n v}(t)-Q_{h y d r o}(t)
$$

where $V\left[\mathrm{~m}^{3}\right]$ is the volume stored in the reservoir, $I\left[\mathrm{~m}^{3} / \mathrm{s}\right]$ is the inflow to the reservoir, $Q_{\text {env }}$ and $Q_{\text {hydro }}$ are the outflows $\left[\mathrm{m}^{3} / \mathrm{s}\right]$ allocated to the river and hydropower plant, respectively. Evaporation and other water losses can easily be introduced as additional terms. For the sake of convenience in illustrating the method and without loss of generality we assume that such terms can be englobed to generate a net inflow $I(t)$. A time step, $\Delta t$, is considered in this study and hence the discretized form of continuity equation is:

$$
V(t+1)=V(t)+\Delta t *\left[I(t)-Q_{e n v}(t)-Q_{h y d r o}(t)\right]
$$

In this work, we consider daily time steps, i.e., $\Delta t=1$. The flow redistribution rules proposed in this study for dammed systems are an extension of previously developed water allocation policies for small hydropower plants (Perona et al., 2013, Gorla and Perona, 2013, Razurel et al., 2016). In these prior studies, non-proportional flow releases were found to be more ecologically and economically efficient compared to the other commonly used flow release rules such as constant minimal flows. Considering storage, inflow and hydropower needs, the following nonproportional water allocation to the environment is proposed for dammed systems:

$$
Q_{e n v}= \begin{cases}Q_{m f r} & I<I_{\min } \\ f_{f e r m i}(I) \cdot f_{s}(V) \cdot\left(I-I_{\min }\right)+Q_{m f r} & I_{\min } \leq I \leq I_{\max } \\ f_{s}(V) \cdot \alpha \cdot \max (I) & I>I_{\max }\end{cases}
$$

where $Q_{m f r}$ is the constant minimal flow release considered compulsory (e.g., as enforced by law), $I_{\min }$ and $I_{\max }$ define the boundaries of streamflow competition (see equation (7)), $f_{\text {fermi }}$ is the Fermi-Dirac function, $f_{s}$ is the storage factor and $\alpha$ determines the magnitude of 
environmental flow. To realize a wide range of possible water allocation policies, we extend the approach of Razurel et al. (2016) to systems with storage. That is, we adopt the mathematical form of the Fermi-Dirac statistical distribution to express the fraction of water allocated to the river $\left(f_{\text {fermi }}\right)$ as a function of inflow. This mathematical distribution is commonly used in quantum statistics to describe a many-particle system in terms of single-particle energy states (Lifshitz and Landau, 1984). The shape of the Fermi-Dirac function depends on only four parameters, which makes it appealing for studying environmental water allocation problems. In order to realize non-proportional environmental flow redistribution rules, we rewrite the FermiDirac function as follows:

$$
f_{\text {fermi }}(I)=\left[1-\left[\left(\frac{Y}{\exp (a(X-b))+c}+M\right)\right]\right] \cdot(j-i)+i
$$

where

$$
\begin{gathered}
M=-\frac{A}{1-A}, \\
A=\frac{\exp (-a \cdot b)+c}{\exp [a \cdot(1-b)+c]}, \\
Y=(1-M) \cdot[\exp (-a \cdot b)+c], \\
X=\frac{I-I_{\min }}{I_{\max }-I_{\min }},
\end{gathered}
$$

where $i, j, a, b$ and $c$ are the parameters that define the shape of the Fermi function. The parameters $i$ and $j$ define the boundaries of the distribution function. When $i<j$, the function monotonously increases and is called the standard Fermi function; when $i>j$, the Fermi function monotonously decreases and is called the inverse Fermi function. The smoothness of the transition between the upper and lower boundaries ( $i$ and $j$ ) is regulated by parameter $a$. A small $a$ results in a linear transition between $i$ and $j$. In contrast, a steeper transition can be realized by increasing $a$. Parameter $b$ sets the location of the inflection point where a value of $b$ between 0 to 
1 can change the location of the inflection point from $I_{\min }$ to $I_{\max }$. Finally, the overall shape of the curve is set by parameter $c$. As far as this work is concerned, parameter $\mathrm{c}$ is set to one. Table 1 shows the range in fermi parameters used in this study to realize a wide range of dynamic environmental flows using non-proportional water allocation rules.

Table 1. The range of Fermi parameters

Fermi parameter
Beginning of the competition
End of the competition
Curvature
Position of the inflection point

$$
\begin{gathered}
\text { Range } \\
0.02 \leq i \leq 0.8 \\
0.02 \leq j \leq 0.8 \\
2 \leq a \leq 8 \\
0 \leq b \leq 1
\end{gathered}
$$

Figure $2 \mathrm{~b}$ illustrates an exemplary visualization of Fermi function defined by equation (4) and (5) while fixing $i$ and $j$ and varying 36 combinations of $a$ and $b$.

Substantially different from no-storage systems (e.g., small hydropower, e.g. see Razurel et al., 2016), here we need to account for effects due to the storage status, which may affect the allocation decision. These effects are accommodated by introducing a storage factor $\left(f_{s}\right)$. We calculate the Relative Stored Water $(R S W)$ in the dam with respect to the storage boundaries $\left(V_{\min }\right.$ and $\left.V_{\max }\right)$ and then the storage factor is calculated using a logistic function (Verhulst, 1845) as:

$$
\begin{gathered}
R S W=\frac{V-V_{\text {max }}}{V_{\text {max }}-V_{\text {min }}}, \\
f_{s}=\frac{L}{1+\exp \left(-k \cdot\left(R S W-x_{0}\right)\right)},
\end{gathered}
$$

where $L$ is the maximum curve value, $k$ determines the curve's steepness and $x_{0}$ is the $\mathrm{x}$-value of the sigmoid curve midpoint. For the purpose of this study, we bound the storage factor between 0 and 1 by defining the logistic parameters as follows: $L=1, k=10$ and $x_{0}=0.5$. From a practical point of view, the storage factor allows to make enough room in the reservoir in order to recover water from flood events while respecting the minimum storage, $V_{\min }$ and maximum storage, $V_{\max }$. This range is regulated by releasing more (less) water to the environment when 
higher (lower) volume of water is stored in the dam. In this way, environmental flows are dynamic even out of the concomitance of flood events and maximum storage, the latter case happening for minimal-flow managed systems. The use of the storage factor associated with nonproportional allocation rules therefore serves as a flood control, limiting the release of high water pulses in a riverine corridor with low hydrological variability. This efficient water management results in a more ecologically friendly water release and reduces the risks associated with floods as mentioned. Notice that the storage factor acts as a dynamic seasonal minimal flow release where a higher summer threshold for minimal flow is usually imposed to ensure sufficient habitat suitability for different species (i.e., fishes). Considering equation (6), the storage factor appears to satisfy this environmental need as higher relative stored water in the dam in summer season results in a higher $f_{s}$.

Finally the ranges of competition for equations (3) are defined as follows:

$$
\begin{gathered}
I_{\min }=Q_{m f r} \\
I_{\max }=\frac{Q_{e n v}^{\max }-Q_{m f r}}{j \cdot f_{s}}+Q_{m f r}
\end{gathered}
$$

where $Q_{e n v}^{\max }$ corresponds to the maximal flow allocated to the environment and is defined as $Q_{e n v}^{\max }=f_{S}(V) \cdot \alpha \cdot \max (I)$. Parameters $\alpha$ and $f_{s}$ determine the magnitude of the maximal environmental flow, and a value of $\alpha=0.3$ is selected for the purpose of this study. Such maximal environmental flow release allows to save water during floods and limits flood related damages. It should be mentioned that $\alpha$ can be regulated to satisfy the environmental needs of every specific site.

\subsection{Environmental indicator}

The environmental suitability of each water allocation policy that releases $Q_{e n v}$ to the environment is evaluated by considering both fish habitat suitability and hydrological indicators.

Fish indicators are of practical use because fishes are an important source of food and can assign an economical benefit of a river status to the neighboring human community. Also, for many fishes habitat requirements are life stage dependent in terms of river morphology and 
hydrodynamics. Furthermore, because of the migration behavior of many species, fish can provide additional information about the longitudinal and lateral connectivity and the passability of a river (Schmutz et al., 1998). In the present study, the fish habitat indicator is defined based on the Weighted Usable Area (WUA) curves of the fishes modeled, for example by use of PHABSIM software (Maddock, 1999, Bloesch et al., 2005). The threshold for the environmental flow rate is defined by the point when fish habitat suitability for fishes rapidly becomes unfavorable. Two thresholds for young and adult fishes are defined where the curvature of the WUA curves is maximized (see Section 3.1). These thresholds were defined on a basis that above a given flow rate the relative environmental benefits for the fishes does not change significantly (Gippel and Stewardson, 1998). Our methodology to assess the fish habitat suitability is inspired by the tool called the Continuous Under Threshold (CUT) habitat duration curves (Capra et al., 1995) where the maximum number of consecutive days below the threshold for young and adult fishes are considered as the most critical period for fish habitat. We follow the same approach but in addition to only considering consecutive days below a threshold, we also calculate the magnitude of the stress period by summing the difference values of WUA for $Q<Q_{\text {threshold }}$ and WUA for $Q_{\text {threshold }}$. We call this Continuous Magnitude Under Threshold (CMUT). Then fish habitat indicators (bounded between 0 and 1) for young and adult fishes are defined based on the maximum value of CMUT as:

$$
\begin{aligned}
& \operatorname{Ind}_{f, y}=1-\frac{\max \left(C M U T_{d, y}\right)-\max \left(C M U T_{n, y}\right)}{\max \left(C M U T_{d, y}\right)+\max \left(C M U T_{n, y}\right)^{\prime}} \\
& \text { Ind }_{f, a}=1-\frac{\max \left(C M U T_{d, a}\right)-\max \left(C M U T_{n, a}\right)}{\max \left(C M U T_{d, a}\right)+\max \left(C M U T_{n, a}\right)},
\end{aligned}
$$

where $d$ and $n$ indices indicate the river flow rate downstream and upstream of the dam, respectively. Furthermore, $y$ and $a$ represent the young and adult fishes. Finally, the geometric mean is used to integrate young and adult fish indicators into a global fish indicator:

$$
\operatorname{Ind}_{f i s h}=\sqrt{\operatorname{Ind}_{f, y} \cdot \operatorname{Ind} d_{f, a}}
$$

Hydrological regimes play an important role in characterizing riparian ecosystems. Efficient ecosystem management can be realized by good understanding hydrologic alteration due to 
human activities. In this study, the extent of hydrologic change for every water allocation policy is based on the methodology proposed by Richter et al. $(1996,1997)$ called the Indicators of Hydrologic Alteration (IHA). The IHA is based on analyzing flow rate and consists of five groups (Table 2): Magnitude timing (1), Magnitude duration (2), Timing (3), Frequency duration (4), Rates of changes frequency (5). The Rate of non Attainment (RnA) and Coefficient of Variation (CV) for 32 IHA are calculated for post (downstream of water intake) and pre (upstream of water intake) impact flow rates. RnA is defined as the fraction of years in which each indicator falls outside the plus and minus one standard deviation around the mean and CV is the ratio of standard deviation to mean in each year. These RnAs and CVs characterize hydrological changes by measuring the number of times and quantity the flow regime is below/above a certain threshold (plus/ minus one standard deviation around the mean) (Gorla and Perona, 2013). However, it should be noted that because we are removing water from the river, which is inevitable due to the hydropower consumption and storage, the benefit of the absolute magnitude of flow regime is not captured. Nonetheless, we believe that considering RnAs and CVs can provide a good understanding of the river hydrological changes due to installation of hydropower systems, especially variability of the flow regime. The latter is an important aspect of the flow regime because of the inconsistencies associated with the current imposed flow regulations (i.e., MFR) in many hydropower systems which has caused several environmental shortcomings, such as reduced the ecosystem biodiversity. Furthermore, the mean squared distance between the pre and post impact RnAs and CVs are calculated (Bizzi et al., 2012). Ultimately, the global hydrological $\left(\operatorname{Ind}_{\text {hydro }}\right.$ ) indicator is found by aggregating and averaging, as detailed in Razurel et al. (2016).

Finally the global environmental indicator is calculated by geometrically averaging the fish habitat and hydrological indicators as follows:

$$
\operatorname{Ind}_{\text {env }}=\sqrt{\operatorname{Ind}_{\text {fish }} \cdot \operatorname{Ind}_{\text {hydro }}}
$$

It should be noted that the choice of defining a single environmental indicator is because it can explicitly show the environmental impact of flow release policies. This way of considering the environmental indicator is more understandable for the community and reservoir operators. 
Furthermore, all the 66 indicators defined in this study are saved and analyzed for a detailed environmental assessment of flow release policies.

Table 2. Summary of hydrological parameters used in the indicators of hydrologic alteration and their characteristics

\begin{tabular}{|c|c|c|}
\hline IHA statistics group & $\begin{array}{l}\text { Regime } \\
\text { characteristics }\end{array}$ & Hydrological parameters \\
\hline Group 1: Magnitude of monthly water conditions & $\begin{array}{l}\text { Magnitude } \\
\text { timing }\end{array}$ & Mean value for each calendar month \\
\hline $\begin{array}{l}\text { Group 2: Magnitude and duration of annual extreme } \\
\text { water conditions }\end{array}$ & $\begin{array}{l}\text { Magnitude } \\
\text { duration }\end{array}$ & $\begin{array}{l}\text { Annual minima 1-day means } \\
\text { Annual maxima 1-day means } \\
\text { Annual minima 3-day means } \\
\text { Annual maxima 3-day means } \\
\text { Annual minima 7-day means } \\
\text { Annual maxima 7-day means } \\
\text { Annual minima 30-day means } \\
\text { Annual maxima 30-day means } \\
\text { Annual minima 90-day means } \\
\text { Annual maxima 90-day means }\end{array}$ \\
\hline $\begin{array}{l}\text { Group 3: Timing of annual extreme water } \\
\text { conditions }\end{array}$ & Timing & $\begin{array}{l}\text { Julian date of each annual 1-day maximum } \\
\text { Julian date of each annual 1-day minimum }\end{array}$ \\
\hline Group 4: Frequency and duration of high/low pulses & $\begin{array}{l}\text { Frequency } \\
\text { duration }\end{array}$ & $\begin{array}{l}\text { No. of high pulses each year } \\
\text { No. of low pulses each year } \\
\text { Mean duration of high pulses within each year } \\
\text { Mean duration of low pulses within each year }\end{array}$ \\
\hline $\begin{array}{l}\text { Group 5: Rate/frequency of water condition } \\
\text { changes }\end{array}$ & $\begin{array}{l}\text { Rate of } \\
\text { changes } \\
\text { frequency }\end{array}$ & $\begin{array}{l}\text { Means of all positive differences between } \\
\text { consecutive daily values } \\
\text { Means of all negative differences between } \\
\text { consecutive daily values } \\
\text { No. rises } \\
\text { No. falls }\end{array}$ \\
\hline
\end{tabular}

\subsection{Optimization method}

In this study, we use multiobjective evolutionary algorithms (MOEAs) to find the Pareto's frontier of the water allocation problem. That is, we search the optimal Fermi parameters $(i, j, a$, $b)$ of Pareto optimal water allocation policies, which ensures the most efficient ecologicaleconomical management. Here, we briefly summarize this methodology.

MOEAs are inspired by the mechanism that biological organisms evolve and transfer their characteristics to their offspring. Form a mathematical point of view, MOEAs are stochastic, direct and population based optimization methods aimed at finding the optimal solutions for complex problems without trivial analytical solutions. The term stochastic refers to the use of 
random operators to search the solution space. It is direct because the fitness of a solution is evaluated by using the value of an objective function and not its derivatives. It is also population based, which means that in every generation a number of potential solutions represent the behavior of the solution space.

MOEAs generate an initial random population and let them evolve to optimal solutions where fitter solutions have a higher chance to survive and reproduce. The evolutionary process is usually performed by applying two main filtering operators: crossover and mutation. The selection methodology is known as roulette wheel, where the solutions with higher fitness are more likely to be selected and evolved. In this study, we benchmark two state of the art MOEAs (NSGA II and Borg MOEA) to build the Pareto's frontier. NSGA II (Deb et al., 2002) is a relatively static MOEA which has been extensively used in the literature. In contrast, Borg MOEA (Hadka and Reed, 2013) is a self-adaptive MOEA which has been found by some recent studies to be efficient in finding efficient operational rules for reservoir systems (e.g., Salazar et al., 2016). An assessment of the quality of the Pareto's frontier, and its associated computational cost, can be made by comparing the results from these two methods. In the following, we briefly review these methods.

NSGA II (Deb et al., 2002) is a fast and elitist MOEA which has been extensively used as an efficient tool for solving multiobjective problems. It features a fast nondominated sorting methodology by calculating a domination count and a set of solutions which dominate each solution. For every generation, nondominated solutions are sorted by comparing both current population and previously found best nondominated solutions. This sorting avoids the chance of losing elite solutions which also results in a faster and more efficient convergence. Furthermore, along with the convergence to Pareto's frontier, it is desired to ensure diversity so as to have a wide spread in the optimal set. NSGA II uses a parameter-less mechanism to maintain diversity in the Pareto's frontier. Furthermore, efficient tuning of NSGA II operators significantly affects its successful convergence to the optimal solution (Salazar et al., 2016). As far as this study is concerned, optimal values for mutation and crossover probability were found to be 0.1 and 0.9 , respectively.

The self-adaptive Borg MOEA (Hadka and Reed, 2013) provides robust optimization by proposing several novel features as well as incorporating design components of other MOEAs. 
Convergence and diversity of Pareto's frontier are ensured using $\epsilon$-dominance archives. $\varepsilon$ progress as a computationally efficient measure of search progression and stagnation is also used. In the case of low convergence speed and search stagnation, randomized restarts are triggered. The latter revives the search by diversifying and resizing the population while preserving selection pressure. Furthermore, to enhance the search domain, Borg incorporates multiple recombination operators and automatically adapts their use based on their relative performance.

To summarize, the procedure of the DPS proposed in this study is shown in Figure 2 where decision variable, objective functions and constraints are defined as follows:

Decision variables: Fermi parameters $(i, j, a$ and $b)$

Objective functions: Environmental indicator (habitat+hydrology) and power production

Physical constraint: reservoir boundaries $\left(V_{\min }\right.$ and $\left.V_{\max }\right)$

Operational constraint: $Q_{\max }, I_{\max }$ and the pattern of energy production. 


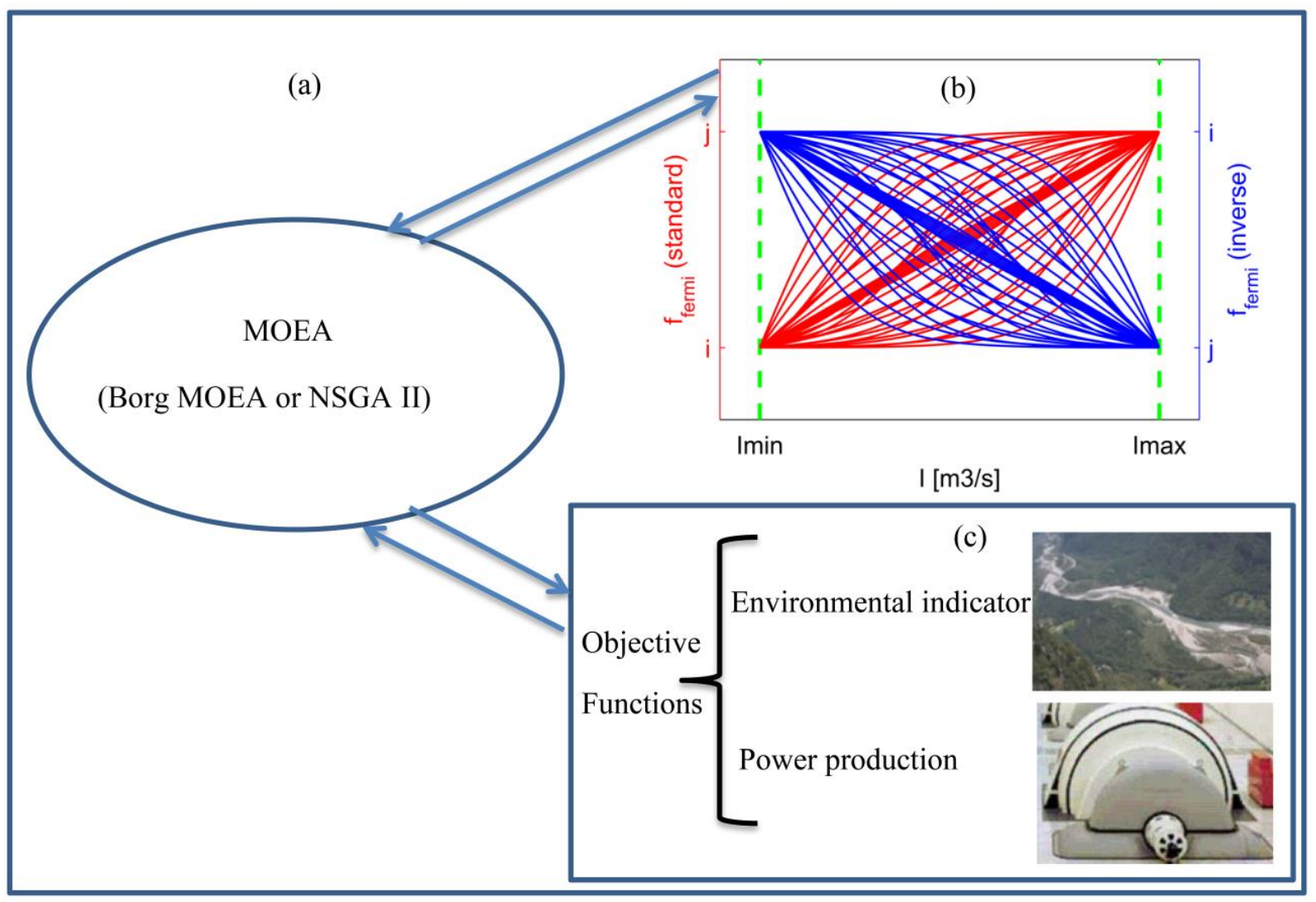

Figure 2. (a) DPS framework (b) 72 Exemplary visualization of fermi function input variables $(\boldsymbol{i}, \boldsymbol{j}, \boldsymbol{a}$ and $\boldsymbol{b})$ while fixing $\boldsymbol{i}$ and $\boldsymbol{j}$ and varying $\boldsymbol{a}$ and $\boldsymbol{b}$. Red curves show standard Fermi functions $(\boldsymbol{i}<\boldsymbol{j})$ and blue curves represent inverse Fermi functions $(\boldsymbol{i}>\boldsymbol{j})$. (c) Objective functions.

\section{Results for a synthetic case and discussion}

\subsection{Generation of synthetic data}

In this section, our methodology is applied to a synthetic case study. First, we build a synthetic natural flow regime (Figure 3a) by rescaling the daily river discharge of the Maggia River located in southeast Switzerland, which is available for the pre-dam period (1929 to 1954). Then, we determine a possible reservoir storage size and hydropower nominal flowrate using the common integral method. The flow duration curve is used to define minimal flow requirement $\left(Q_{m f r}=0.18 \mathrm{~m}^{3} / \mathrm{s}\right.$ and $\left.Q_{2 m f r}=0.21 \mathrm{~m}^{3} / \mathrm{s}\right)$. In this way, the reservoir available storage, $V_{\max }$ is set to $41 \mathrm{Mm}^{3}$, and a sensitivity analysis for $V_{\max }$ will later be performed to evaluate the effect of uncertainties on the choice of reservoir size. For the sake of simplicity to illustrate the basic 
ideas of our methodology, we consider weekly periodic flowrate demands corresponding to the nominal turbine capacity where turbines operate only in the working days which are assigned to the hydropower as a first priority based on the available storage in the reservoir (Figure 3b).

(a)

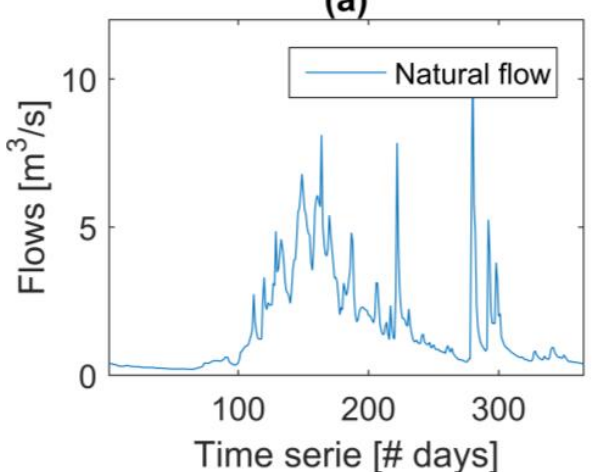

(c)

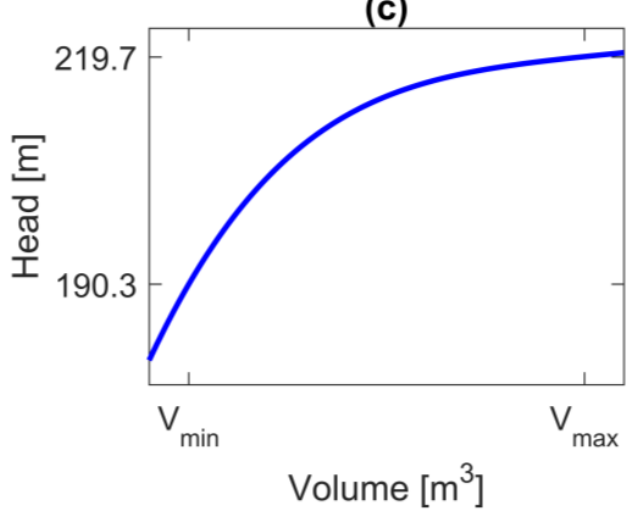

(b)

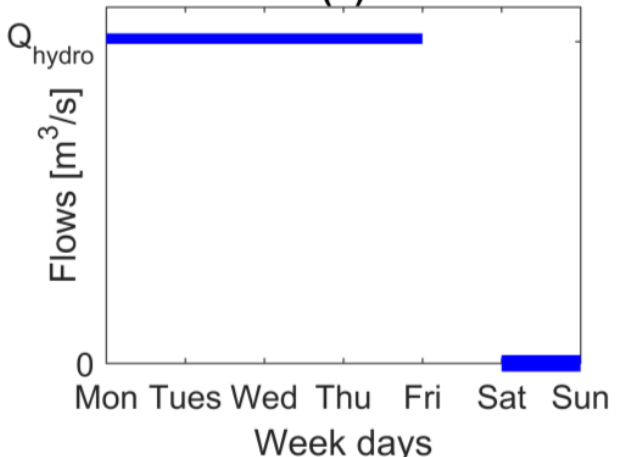

(d)

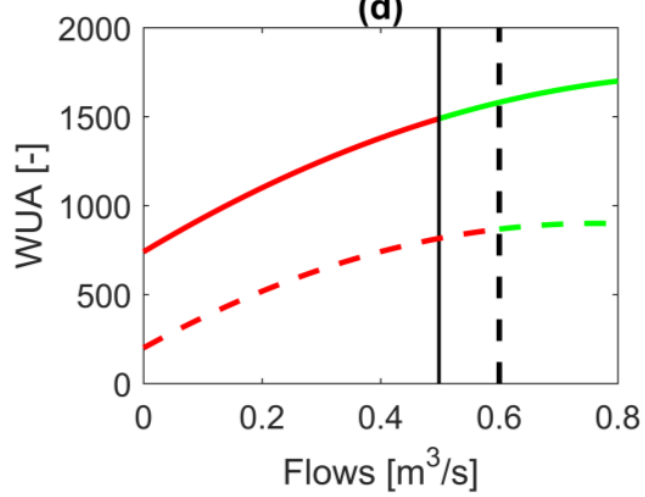

Figure 3. (a) Natural flow regime. (b) Weekly hydropower flowrate demand. (c) Reservoir's head-volume relationship. (d) WUA curves for young (solid line curve) and adult fishes (dashed line curve). Vertical lines denote the assigned thresholds based on the WUA curves. The green and red colors represent the flow rates in which their associated WUA are higher and lower than the threshold, respectively.

Energy production is computed using the following storage-dependent relationship:

$$
P=\rho \cdot Q_{h y d r o} \cdot g \cdot H(V) \cdot \frac{24}{10^{6}}[\mathrm{MWh}]
$$

where $\rho$ and $g$ are water density and gravity, respectively. $H$ is the reservoir water level, which is assumed to be a polynomial function of the storage (Figure 3c). Furthermore, Figure 3d shows the WUA curves considered in this study to calculate fish habitat indicators for both young and adult fishes. The results of our methodology are compared with other simulated policies, which 
are constant (one and two threshold) minimum flows $\left(Q_{m f r}\right.$ and $\left.Q_{2 m f r}\right)$, and proportional releases by assigning fixed percentages (from $1 \%$ to $15 \%$ ) of the inflow.

\subsection{Pareto frontier and optimal water allocation}

Figure 4a shows the global efficiency plot resulting from adopting optimal non-proportional redistribution rules based on the Fermi functions and other proportional and MFR policies. Notably, an almost vertical (Pareto optimal) frontier where energy production is maximal can be identified. This is an important result because it shows that DEF releases via non-proportional redistribution rules guarantees better global efficiency of water storage system compared to policies applying constant minimum and proportional flow. The significant improvement in the ecological indicator at almost the same energy production is seen to arise precisely from the reservoir storage dynamic. Furthermore, as discussed in Section 2.3, NSGA II performance is dependent on parameters tuning. As shown in Figure 4, the Pareto's frontier simulated with Borg MOEA is the same as the one obtained with NSGA II. This indicates that the NSGA II parameters have been efficiently tuned. Also, it should be mentioned that in terms of running time, Borg MOEA used almost half the time as NSGA II to find the Pareto's frontier. This reveals the fact that using an adaptive optimization approach can substantially speed up the optimization process. 


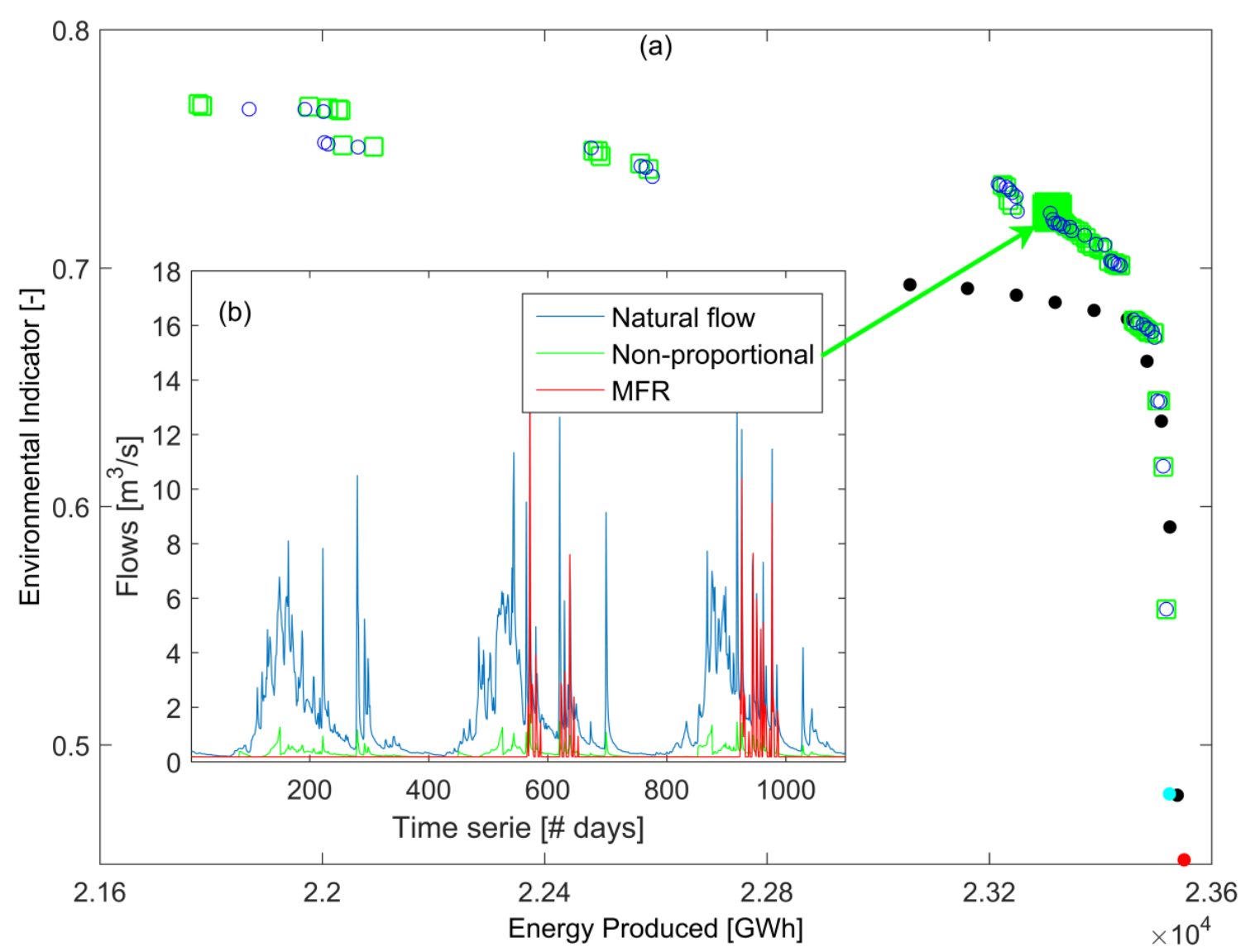

Figure 4. (a) Pareto's frontier and alternative scenarios (minimal flow release and proportional release). Blue circles and green squares represent the scenarios located on the Pareto's frontier obtained with NSGA II and Borg MOEA, respectively. Black, cyan and red dots denote the proportional, seasonal MFR and MFR flow release policies, respectively. The bold green square is selected as an exemplary non-proportional flow release rule from Pareto's frontier and hereafter we perform some detailed analysis which can help for further evaluation and comparison between different flow release rules. The followings characterize the fermi parameters of this non-proportional flow release rule: $i=0.11, j=0.04, a=7.4, b=0.98$. (b) Hydrographs corresponding to different flow release rules.

Through non-proportional water allocation, the imposed flow releases create enough room in the reservoir to allow to capture and laminate flood events while recovering part of them for energy production. This is clearly seen by comparing the hydrograph resulting from applying the nonproportional flow release policy with that obtained for constant minimal flow (Figure 4b). Notably, although the quantities of water allocated in both policies are almost the same, the variability arising from non-proportional redistribution results in a more ecologically sustainable streamflow. From an ecological perspective such variability is indeed important to maintain transversal connectivity between the channel and floodplain, which occurs with a frequency comparable to the natural one. 
Figure 5 shows the simulated daily volume of stored water in the reservoir resulting from both non-proportional and constant minimal flow requirement water allocation policies. As shown in this figure, an efficient reservoir storage dynamic policy allows for better environmental and economic efficiency. This dynamic behavior in reservoir storage is mainly due to the use of storage factor in the non-proportional flow release policy, which enables for a more efficient water management. The efficient use of dynamic storage creates flow variability similar to natural flow by making enough room in the reservoir to capture and laminate flood events. The use of the storage factor is an alternative to the traditional way of managing water in dammed systems where a constant minimal flow is always allocated to the environment unless for the time when the maximum storage level in the reservoir is reached. In that case, the overflow must be also released to the river. On the one hand, in extreme conditions such releases may combine with flooding, which may harm urban areas and endanger human lives. Therefore, the storage factor allows to laminate the release of high water pulses during flooding events. As far as our synthetic case is concerned, non-proportional rules decrease the number of days corresponding to flood release due to reservoir overflow by approximately $75 \%$ compared to minimal flow policy (Figure 4). Furthermore, the ecological impacts of floods are vital for some riparian processes involving vegetation and transport phenomena in general (Džubáková et al., 2015). The dynamic flow release resulting from non-proportional water allocation policies can meet such environmental needs and enforce the release of higher flow pulses at the time of occurrence. 


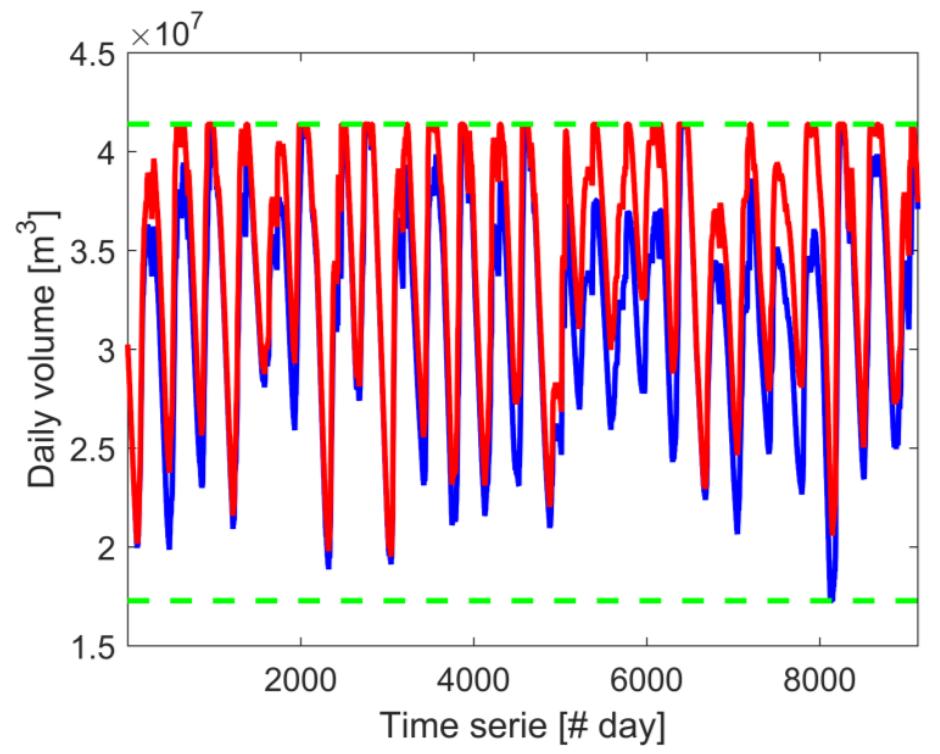

Figure 5. Comparison of the simulated daily volume of stored water in the reservoir. Blue curve denote the nonproportional flow release and red curve represents the constant minimal flow requirement water allocation policy. Green dashed lines show physical boundaries of the reservoir $\left(V_{\max }\right.$ and $\left.V_{\min }\right)$

Figure 6 shows the comparison between the natural regime (green line), constant minimum flow (red line) and non-proportional flow release (blue line) for three exemplary IHA corresponding to three groups of Richter's hydrological indicators (i.e., 3, 4 and 5 from Table 2) representing flow variability. Non-proportional flow redistribution rules impact less on the natural flow regime compared to constant minimal flow water allocation policy. This environmental amelioration is significant when the Julian date (JD) of each annual 1-day maximum is considered (Figure 6a). This indicator describes the importance of the timing occurrence of high extreme water conditions within an annual cycle. A comparison of the impact of flow regime and timing provides a mechanism for evaluating if requirements for specific life-cycles are satisfied, the degree of mortality or stress related to extreme water conditions, such floods. As shown in this figure, the minimal flow release rule strongly offsets the annual timing of high events from the natural flow regime. This improvement in environmental efficiency is also seen when indicators of groups 4 and 5 are considered. These indicators describe flow variability based on the flow regime in terms of frequency, duration and rate of change of the flow regime. The time duration that a certain water condition lasts can determine if a particular life-cycle phase can be completed or the extent of a stressful period can accumulate. Furthermore, the rate of change in a water condition can be used as a measure to characterize the rate and frequency of inter-annual environmental change (Richter et al., 1996). Figure 6b and Figure 6c show two exemplary 
indicators from groups 4 and 5, which are the number of high pulses each year and number of rises, respectively. These indicators clearly show that the variability arising from a nonproportional water release policy can enable significant environmental improvements. The CVs and $\mathrm{RnAs}$ for different flow release rules compared with the natural flow regime confirm these environmental benefits. As an example, Table 3 compares the simulated RnAs and CVs corresponding to the number of rising indicators (Figure 6c) under different flow regimes.

(a)

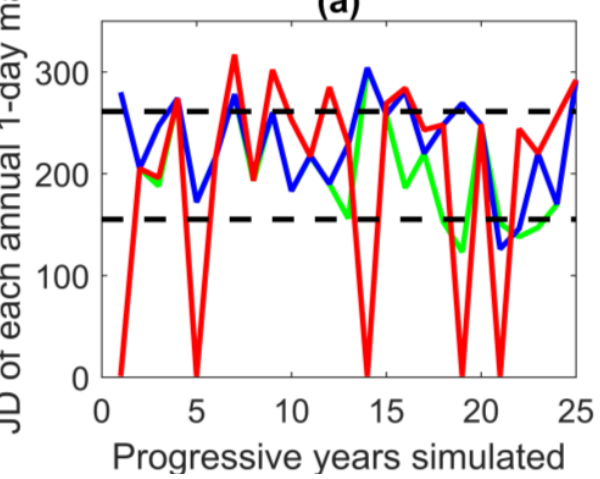

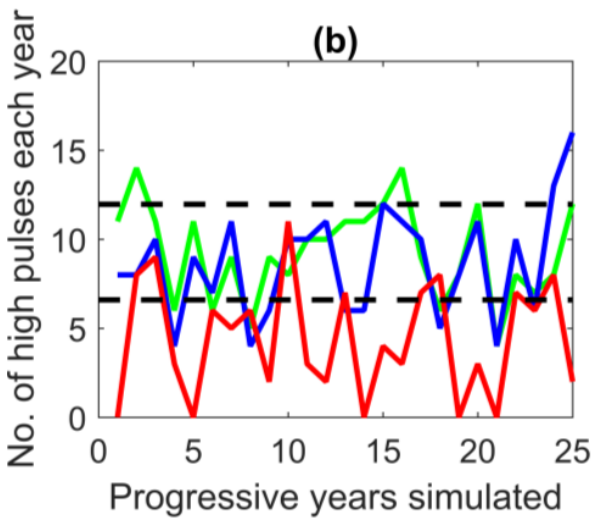

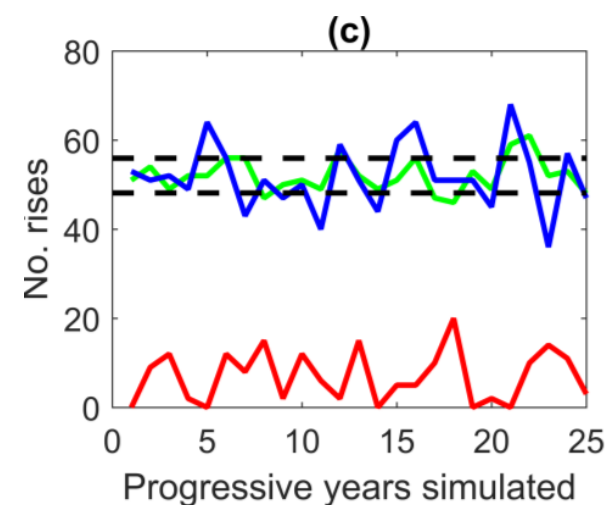

Figure 6. Comparison of three selected IHA corresponding to three groups between the natural regime (green line), constant ninimum flow (red line) and non-proportional flow release (blue line). Dashed lines define $\pm \boldsymbol{S D}$ around the mean of the natural egime IHA.

Table 3. Comparison of the simulated RnAs and CVs belonging to the number of rises indicator between the natural regime, constant minimum flow and non-proportional flow release

$\begin{array}{cccc} & \text { Natural flow regime } & \text { Non-proportional } & \text { Minimal flow requ } \\ \text { RnA } & 0.4 & 0.6 & 1 \\ \text { CV } & 0.07 & 0.12 & 0.84\end{array}$

\subsection{Influence of reservoir storage and river hydrology}

We now investigate the impact of storage size on dam ecological-economical efficiency under the assumption that our design for reservoir size in the synthetic case was conservative. We perform a sensitivity analysis where we vary the maximum storage size of the dam in the range $0.9 V_{\max } \div 1.4 V_{\max }$. Figure 7 shows that increasing the storage size allows for better environmental and economical (Pareto) efficiencies up to a certain storage size (i.e., in this case 
$\sim 1.3 V_{\text {max }}$ ), as expected. This value corresponds to the reservoir volume that allows to capture and best allocates all the incoming water under the assigned hydrologic/climatic and energy production conditions. Furthermore, it should be mentioned that the energy production, corresponding to the vertical part of Pareto's frontier, slightly increases (1.8\%) when the reservoir size changes from $V_{\max }$ to $1.3 V_{\max }$.

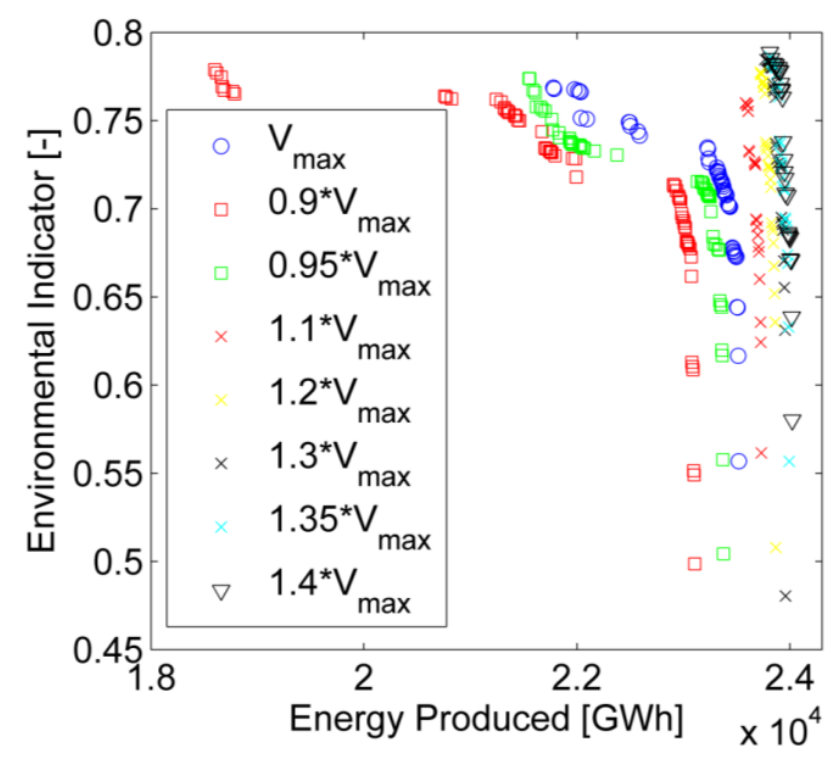

Figure 7. Maximum reservoir storage size sensitivity analysis

Another important variable that may influence the Pareto's frontier shape is the variability of the natural flow regime. To this purpose, we generate 100 random hydrological regimes by shuffling the 25 years of inflow data annually and investigating the change in the efficient frontier. While performing this shuffling process, the linear statistics of the inflow signals remains the same, thus preserving the catchment dynamics. Figure 8a shows the simulated Pareto's frontiers resulting from all 100 hydrological regimes. In the lower-right side of the figure, the flow release policies are similar to constant minimal flow policies where less diversity is observed in the Pareto's frontier shape. Hence, when less water is allocated to the environment, the ecologicaleconomic efficiency is less dependent on that particular hydrological regime. However, the Pareto's frontier shape is more sensitive to hydrological regimes when more water is released to the environment. This can be seen in the top-left side of the figure where the Pareto's frontier shapes are more dispersed. Furthermore, from these Pareto's frontiers, non-dominated scenarios (red squares in the figure) can be selected from the most efficient both economical and 
environmentally friendly flow release policies under different hydrological regimes as described. Therefore, we can investigate the performance of these specific efficient scenarios when they are operated with the same 100 random hydrological regimes. Flow release rules that are less dependent on hydrological regimes are more appealing because they can still perform efficiently under hydrological changes. In that respect, we consider only those scenarios that are expected to be less dependent on the flow regime (nondominated scenarios which have energy production more than $2.3 * 10^{4} \mathrm{GWh}$ in Figure $8 \mathrm{a}$ ). As it can be seen in Figure $8 \mathrm{~b}$, these selected flow release rules still show efficient environmental and economic performances when they operate under different hydrological regimes. In particular these non-proportional flow release rules guaranty better global efficiency under different hydrological regimes compared to minimal flow release policies.

(a)

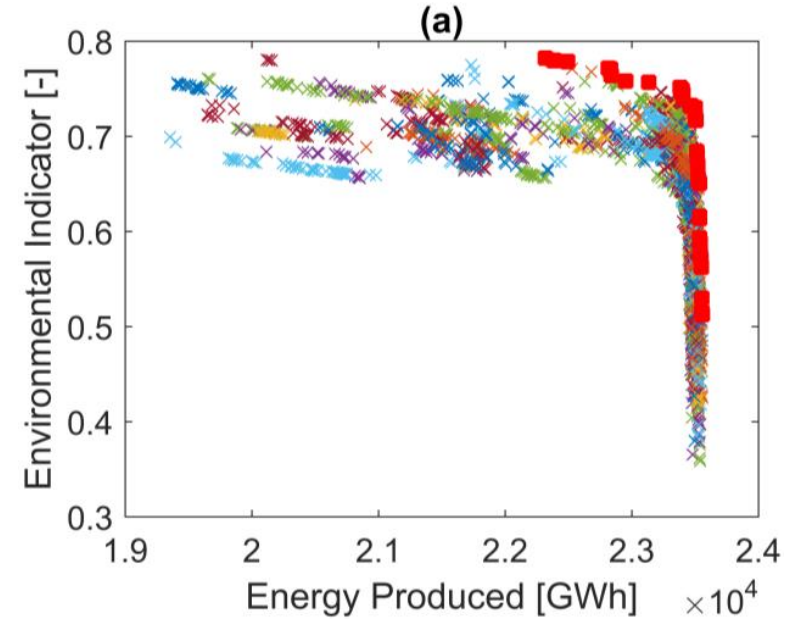

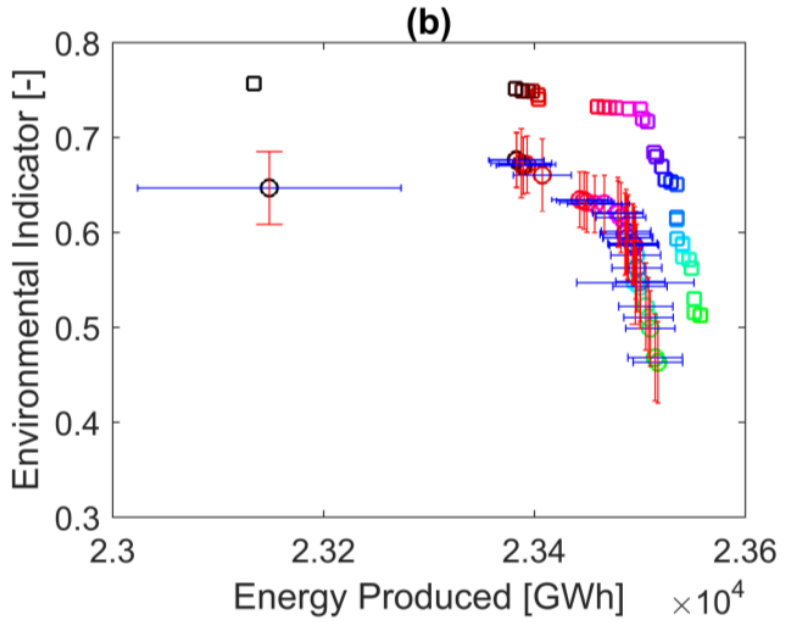

Figure 8. The impact of hydrological changes on the shape of Pareto's frontier: (a) comparison of the simulated Pareto's frontiers resulting from 100 random hydrological regimes. Every color represents a Pareto's frontier and red squares denote to nondominated scenarios among all the Pareto's frontiers. (b) Evaluation of the selected flow release rules (squares) performances under random hydrological regime changes. Symbols with the same color represent the calculated energy production and environmental indicator with the same flow release rule. Circles denote the mean environmental and economical efficiencies simulated with 100 hydrological regimes; horizontal and vertical error bars represent $\pm S D$ around the mean of the simulated power productions and environmental indicators, respectively.

The results shown here are promising, although we stress that implementing non-proportional redistribution rules in existing power plants should be carefully evaluated. For example, for power plants that are already capable of storing all incoming flows and laminate all flooding, it 
may not be possible to improve the environmental indicator at equal energy production. In particular, this should be done in relation to specific river hydrologic regime, size of the actual dam and the flexibility of intakes that impound the surrounding water courses. This requires additional and more thorough numerical analyses, as well as an evaluation of the environmental benefits, by means of case by case specific indicators.

\section{Conclusions}

We make use of two MOEAs (NSGA II and Borg MOEA) and compare their relative performance in our DPS framework to build Pareto's frontier. The results suggest that nonproportional flow releases provide a broader spectrum of globally-efficient performances of the whole system (i.e., hydropower plus environment) compared to constant minimum flow release operational policies. More explicitly, a vertical Pareto's frontier in the global efficiency plot means that substantial improvement in the environmental indicator can be achieved without inducing a significant loss in energy production. This result can be realized by engineering new (i.e., non-proportional) dynamic environmental flow release policies. Such an improvement is found to be mainly due to a better use of reservoir storage dynamics, which enables to capture and laminate flood events while recovering part of them for energy production. Although not for all, these changes could bring substantial improvement to hydropower systems with specific basin soil and hydrological characteristics. Regarding reservoir size, it was shown that Pareto solutions maintain a vertical frontier over a reasonable storage size range, which offers some design flexibility. The Pareto's frontier shape under different hydrological regimes was also assessed, indicating that non-proportional flow releases remain efficient also under uncertainties of the hydrological statistics.

\section{Acknowledgments}

We thank the Swiss National Science Foundation for funding the projects NFP70 HydroEnv (Grant No. 407040153942/1) and REMEDY (Grant No. PP00P2153028/1). The SCCER-SoE is also acknowledged for both scientific and financial support. 


\section{References}

ARTHINGTON, A. H., BUNN, S. E., POFF, N. L. \& NAIMAN, R. J. 2006. The challenge of providing environmental flow rules to sustain river ecosystems. Ecological Applications, 16, 1311-1318.

ASSANI, A. A., QUESSY, J.-F., MESFIOUI, M. \& MATTEAU, M. 2010. An example of application: The ecological "natural flow regime" paradigm in hydroclimatology. Advances in Water Resources, $33,537-545$.

BARTHOLOW, J. M. 2010. Constructing an interdisciplinary flow regime recommendation1. JAWRA Journal of the American Water Resources Association, 46, 892-906.

BELLMAN, R. 1957. E. 1957. dynamic programming. Princeton UniversityPress. BellmanDynamic programming1957, 151.

BIZZI, S., PIANOSI, F. \& SONCINI-SESSA, R. 2012. Valuing hydrological alteration in multi-objective water resources management. Journal of Hydrology, 472, 277-286.

BLOESCH, J., SCHNEIDE, M. \& ORTLEPP, J. 2005. An application of physical habitat modelling to quantify ecological flow for the Rheinau hydropower plant, River Rhine. Archiv für Hydrobiologie. Supplementband. Large rivers, 16, 305-328.

CAPRA, H., BREIL, P. \& SOUCHON, Y. 1995. A new tool to interpret magnitude and duration of fish habitat variations. Regulated rivers: research \& management, 10, 281-289.

CASTELLETTI, A., PIANOSI, F. \& SONCINI-SESSA, R. 2008. Water reservoir control under economic, social and environmental constraints. Automatica, 44, 1595-1607.

CUI, L. \& KUCZERA, G. 2005. Optimizing water supply headworks operating rules under stochastic inputs: Assessment of genetic algorithm performance. Water Resources Research, 41.

DARIANE, A. B. \& MOMTAHEN, S. 2009. Optimization of multireservoir systems operation using modified direct search genetic algorithm. Journal of Water Resources Planning and Management, 135, 141-148.

DEB, K., PRATAP, A., AGARWAL, S. \& MEYARIVAN, T. 2002. A fast and elitist multiobjective genetic algorithm: NSGA-II. Evolutionary Computation, IEEE Transactions on, 6, 182-197.

DŽUBÁKOVÁ, K., MOLNAR, P., SCHINDLER, K. \& TRIZNA, M. 2015. Monitoring of riparian vegetation response to flood disturbances using terrestrial photography. Hydrology and Earth System Sciences, 19, 195-208.

FRANCHINI, M., VENTAGLIO, E. \& BONOLI, A. 2011. A procedure for evaluating the compatibility of surface water resources with environmental and human requirements. Water resources management, 25, 3613-3634.

GIPPEL, C. J. \& STEWARDSON, M. J. 1998. Use of wetted perimeter in defining minimum environmental flows. Regulated rivers: research \& management, 14, 53-67.

GIULIANI, M., CASTELLETTI, A., PIANOSI, F., MASON, E. \& REED, P. M. 2015. Curses, tradeoffs, and scalable management: Advancing evolutionary multiobjective direct policy search to improve water reservoir operations. Journal of Water Resources Planning and Management, 142, 04015050.

GORLA, L. 2014. The riparian environment as a non-traditional water user: experimental quantification and modelling for hydropower management. PhD thesis, EPFL, $n^{\circ} 6314,2014$

GORLA, L. \& PERONA, P. 2013. On quantifying ecologically sustainable flow releases in a diverted river reach. Journal of Hydrology, 489, 98-107.

GUO, X., HU, T., ZENG, X. \& LI, X. 2012. Extension of parametric rule with the hedging rule for managing multireservoir system during droughts. Journal of Water Resources Planning and Management, 139, 139-148. 
HADKA, D. \& REED, P. 2013. Borg: An auto-adaptive many-objective evolutionary computing framework. Evolutionary Computation, 21, 231-259.

HEIDRICH-MEISNER, V. \& IGEL, C. Variable metric reinforcement learning methods applied to the noisy mountain car problem. European Workshop on Reinforcement Learning, 2008. Springer, 136150.

KENNARD, M. J., PUSEY, B. J., OLDEN, J. D., MACKAY, S. J., STEIN, J. L. \& MARSH, N. 2010. Classification of natural flow regimes in Australia to support environmental flow management. Freshwater Biology, 55, 171-193.

KERN, J. D., CHARACKLIS, G. W., DOYLE, M. W., BLUMSACK, S. \& WHISNANT, R. B. 2011. Influence of deregulated electricity markets on hydropower generation and downstream flow regime. Journal of Water Resources Planning and Management, 138, 342-355.

KONAR, M., TODD, M. J., MUNEEPEERAKUL, R., RINALDO, A. \& RODRIGUEZ-ITURBE, I. 2013. Hydrology as a driver of biodiversity: Controls on carrying capacity, niche formation, and dispersal. Advances in Water Resources, 51, 317-325.

LAZZARO, G., BASSO, S., SCHIRMER, M. \& BOTTER, G. 2013. Water management strategies for run-ofriver power plants: Profitability and hydrologic impact between the intake and the outflow. Water Resources Research, 49, 8285-8298.

LIFSHITZ, E. \& LANDAU, L. 1984. Statistical Physics (Course of Theoretical Physics, Volume 5). Butterworth-Heinemann.

MADDOCK, I. 1999. The importance of physical habitat assessment for evaluating river health. Freshwater Biology, 41, 373-391.

MEIJER, K., VAN DER KROGT, W. \& VAN BEEK, E. 2012. A new approach to incorporating environmental flow requirements in water allocation modeling. Water resources management, 26, 1271-1286.

MOYLE, P. B. \& MOUNT, J. F. 2007. Homogenous rivers, homogenous faunas. Proceedings of the National Academy of Sciences, 104, 5711-5712.

OLIVEIRA, R. \& LOUCKS, D. P. 1997. Operating rules for multireservoir systems. Water Resources Research, 33, 839-852.

PERONA, P., DÜRRENMATT, D. J. \& CHARACKLIS, G. W. 2013. Obtaining natural-like flow releases in diverted river reaches from simple riparian benefit economic models. Journal of environmental management, 118, 161-169.

PETTS, G. E. 1996. Water allocation to protect river ecosystems. Regulated rivers: research \& management, 12, 353-365.

PETTS, G. E. 2009. Instream flow science for sustainable river management1. Wiley Online Library.

POFF, N. L., RICHTER, B. D., ARTHINGTON, A. H., BUNN, S. E., NAIMAN, R. J., KENDY, E., ACREMAN, M., APSE, C., BLEDSOE, B. P. \& FREEMAN, M. C. 2010. The ecological limits of hydrologic alteration (ELOHA): a new framework for developing regional environmental flow standards. Freshwater Biology, 55, 147-170.

POWELL, W. B. 2007. Approximate Dynamic Programming: Solving the curses of dimensionality, John Wiley \& Sons.

RAZUREL, P., GORLA, L., CROUZY, B. \& PERONA, P. 2016. Non-proportional Repartition Rules Optimize Environmental Flows and Energy Production. Water resources management, 30, 207-223.

RICHTER, B. D., BAUMGARTNER, J. V., POWELL, J. \& BRAUN, D. P. 1996. A method for assessing hydrologic alteration within ecosystems. Conservation biology, 1163-1174.

RICHTER, B. D., BAUMGARTNER, J. V., WIGINGTON, R. \& BRAUN, D. P. 1997. How much water does a river need? Freshwater Biology, 37, 231-249.

SALAZAR, J. Z., REED, P. M., HERMAN, J. D., GIULIANI, M. \& CASTELLETTI, A. 2016. A diagnostic assessment of evolutionary algorithms for multi-objective surface water reservoir control. Advances in Water Resources, 92, 172-185. 
SCHMUTZ, S., GIEFING, C. \& WIESNER, C. 1998. The efficiency of a nature-like bypass channel for pikeperch (Stizostedion lucioperca) in the Marchfeldkanalsystem. Hydrobiologia, 371, 355-360.

SCHWEIZER, S., BORSUK, M. \& REICHERT, P. 2007. Predicting the morphological and hydraulic consequences of river rehabilitation. River research and Applications, 23, 303-322.

TA, J., KELSEY, T. R., HOWARD, J. K., LUND, J. R., SANDOVAL-SOLIS, S. \& VIERS, J. H. 2016. Simulation Modeling to Secure Environmental Flows in a Diversion Modified Flow Regime. Journal of Water Resources Planning and Management, 05016010.

TSITSIKLIS, J. N. \& VAN ROY, B. 1996. Feature-based methods for large scale dynamic programming. Machine Learning, 22, 59-94.

VERHULST, P. 1845. La Loi d'Accroissement de la Population. Nouv. Mem. Acad. Roy. Soc. Belle-lettr. Bruxelles, 18, 1.

YEH, W. W. G. 1985. Reservoir management and operations models: A state-of-the-art review. Water Resources Research, 21, 1797-1818. 